\title{
Exact variational principle for 3-D unsteady heat conduction with second sound
}

Gaolian Liu

Institute of Mechanics, Shanghai University, Shanghai, 200072, China

Errata to: Journal of Thermal Science, Vol. 15(2006) 361-363

DOI: 10.1007/s11630-006-0361-7

The list of references of the entitled paper was misprinted and should be corrected as follows:

\begin{tabular}{|l|l|}
\hline \multicolumn{1}{|c|}{ Incorrect } & \multicolumn{1}{c|}{ Correct } \\
\hline [4] Vujanovic, B., An approach to linear and nonlinear & [4] Vujanovic, B., An approach to linear and nonlin- \\
heat transfer problem using a Lagrangian. & ear heat transfer problem using a Lagrangian, AIAA \\
[5] AIAA Journal, 9, No.1 (1971)131-134\{see also: & Journal, 9 (1971), 131-134 \\
ibid, 9, No.2(1971) 327-330\} & Vujanovic, B, Strauss, A.M., Heat transfer with \\
{$[6]$} & nonlinear boundary conditions via a variational prin- \\
{$[7]$} & ciple, AIAA Journal, 9 (1971), 327-330 \\
{$[8]$} & {$[5]$} \\
{$[9]$} & {$[6]$} \\
{$[10]$} & {$[7]$} \\
& {$[8]$} \\
& {$[9]$} \\
\hline
\end{tabular}

On p. 363, two errors should be corrected as follows:

\begin{tabular}{|c|c|}
\hline \multicolumn{1}{|c|}{ Incorrect } & Correct \\
\hline $\begin{array}{c}\text { p. 363, line 15: At the initial }(\mathrm{t}=0) \text { and final }\left(\mathrm{t}_{\mathrm{f}}\right) \\
\text { instances: }\end{array}$ & At the initial $(\mathrm{t}=0)$ and the final $\left(\mathrm{t}_{\mathrm{f}}\right)$ instants: \\
\hline p. 363, Eq.(18), in the last integral: $\left(\frac{\partial T}{\partial t}\right)$ & $\left(\frac{\partial T}{\partial t} T\right) t_{f}$ \\
\hline
\end{tabular}

